\title{
THE INFLUENCE OF THE TOP AND THE BOTTOM GRAIN BOUNDARIES ON THE CURRENT TRANSPORT IN $\mathrm{YBa}_{2} \mathrm{Cu}_{3} \mathrm{O}_{7-\delta}$ STEP-EDGE JOSEPHSON JUNCTION
}

\author{
F. LOMBARDI*†, Z. G. IVANOV, P. KOMISSINSKI†, G. M. FISCHERß, \\ P. LARSSON and T. CLAESON \\ Physics Department, Chalmers University of Technology and Göteborg University, \\ S-41296 Göteborg, Sweden
}

\begin{abstract}
We have studied the electrical transport properties of the grain boundaries (GBs) formed at the top and at the bottom edges of $\mathrm{YBa}_{2} \mathrm{Cu}_{3} \mathrm{O}_{7-\delta}$ step-edge Josephson junctions, for different values of the step angle $\alpha$. The step-edge junctions were fabricated on (100) $\mathrm{LaAlO}_{3}$ steps using a tilted $\mathrm{Ar}$ ion milling. Due to the shadowing effect of the step, the middle and the bottom part of the step edge junction were contacted by thin stripes. We found that for $\alpha \approx 60^{\circ}$ the top GB is responsible for the weak link behaviour of our step-edge junctions. On less steep steps, $\alpha \approx 45^{\circ}$, a series of GBs with weak link properties were found to nucleate along the step profile. We also correlated these results with the different microstructural properties of the GBs formed on steps with different angles. (C) 1999 Elsevier Science Ltd. All rights reserved
\end{abstract}

\section{INTRODUCTION}

Since the discovery of high critical temperature superconductors (HTS) great effort has been devoted to the fabrication of Josephson junctions. They, in fact, represent the backbone of active superconductive electronics. At the same time they can be used as a powerful tool to explore fundamental properties of superconductors [1]. The short coherence length of HTS, together with the microscopic structure of the junction interfaces have, however, imposed severe restrictions to the development of conventional structures based on multilayer technology [2].

The development of grain boundary (GB) Josephson junctions, obtained by the controlled nucleation of grains with different crystallographic orientation, has represented an important innovation. Among the possibilities to produce GB junctions, step-edge junctions (SEJs) are extremely interesting for the simplicity of the fabrication process and for the liberty to place them at will on the substrate. They are formed during the YBCO film growth on a step fabricated in the substrate. The profile of the step is very important as it determines the microstructure of the YBCO film grown on the step and therefore the electromagnetic properties of the device. Depending on the step angle, $\alpha$, and in general on the local presence of step defects [3] it is, in fact, possible to obtain structurally different GBs, at the top and bottom edges and along the step profile [4-6]. Moreover, these GBs are a complex combination of $90^{\circ} c$-axis tilted GBs, namely the symmetrical GB (SGB) and the basal plane faced tilted grain boundary (BPGB) $[5,6]$. This makes it more complicated to separate, from the transport measurements, the contributions of different GBs.

In this paper we characterize the transport properties of the GBs which form a SEJ on a $\mathrm{LaAlO}_{3}$ step, for different values of the step angle. We were able to separate the superconductive properties of the two GBs formed at the top and bottom edges of the step (or of the top GB and of the series of GBs formed eventually along the profile of the step). This was obtained by using, the shadowing effect of the step to protect the material present on it, during a tilted $\mathrm{Ar}$ ion milling etching, in analogy with the work of M. Grove et al. [7]. For steps with $\alpha \approx 60^{\circ}$ this procedure was applied to define the electrode and the microbridges. In this way, due to the sha-

*Author to whom correspondence should be addressed.

†On leave from Department of Physics, University of Napoli "Federico II", I-80125 Napoli, Italy.

\$On leave from Institute of Radio Engineering and Electronics RAS, Moscow 103907, Russia.

§Present address-Department of Physics, Technical University of Denmark, DK-2800 Lyngby, Denmark. 

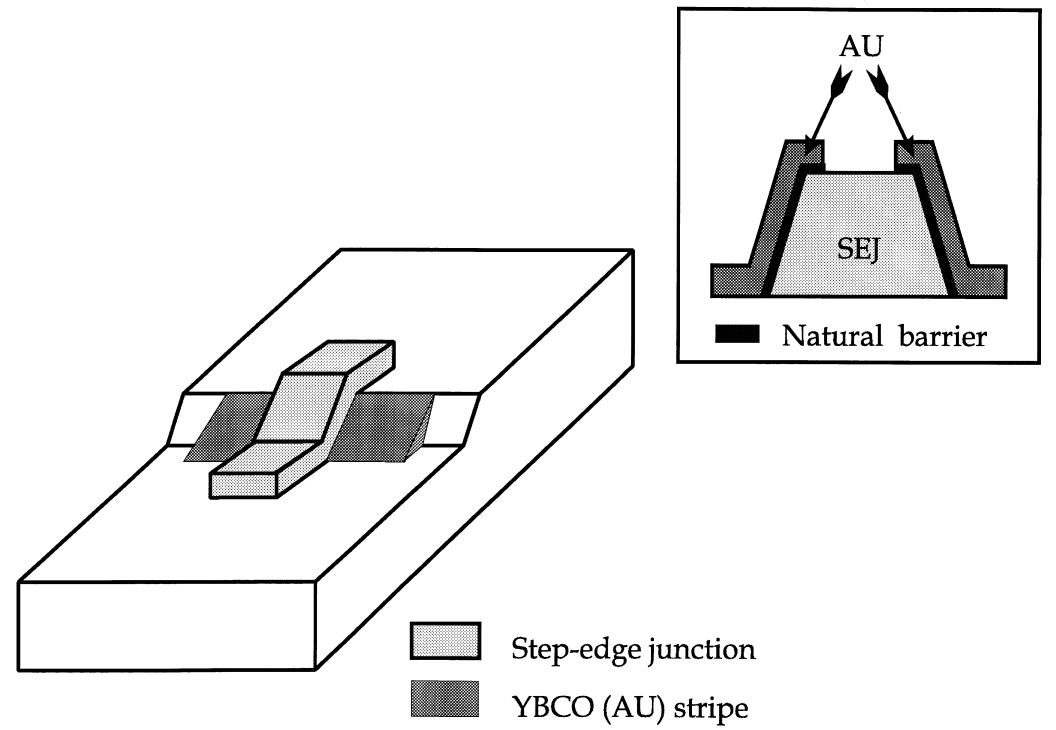

Fig. 1. Schematic view of a step edge junction with the presence of YBCO (Au) stripes along the step. The inset shows a cross section of the devices realized on $45^{\circ}$ steps.

dow of the step, a continuous YBCO film remained along the step enabled the middle and bottom part of the SEJ to be contacted (see Fig. 1). The superconductive properties of the isolated top GB could therefore be compared with those of the whole SEJ. In the case of a less sharp step $\left(\alpha \approx 45^{\circ}\right)$ SEJs were first defined. An Au film, subsequently deposited, was patterned using the tilted Ar ion milling technique and remained along the step and in contact with the middle and bottom part of the microbridge (see the inset of Fig. 1). This second procedure was developed for the possibility, that it provides, to study non equilibrium effects in HTS. The interface $\mathrm{Au} / \mathrm{YBCO}$ can, in fact, be used as a natural barrier for injecting quasiparticles in the GB region and in its surroundings [8].

\section{FABRICATION PROCEDURE}

In this work an amorphous carbon (a-C) mask and e-beam lithography have been used for the fabrication of the step, about $250-270 \mathrm{~nm}$ high, in a $\mathrm{LaAlO}_{3}$ substrate.

A $200 \mathrm{~nm}$ thick a-C film was deposited on a (100) $\mathrm{LaAlO}_{3}$ substrate by a plasma deposition procedure using $\mathrm{CH}_{4}$ as process gas. A $50 \mathrm{~nm}$ thick Au film was then deposited by electron beam evaporation on the a-C film. The geometry of the step was defined using a direct electron beam writing on a SAL 601 negative resist. Ion beam etching perpendicular to the substrate at a beam voltage, $V_{\text {beam }}=500 \mathrm{~V}$, and a beam current density, $J=0.2 \mathrm{~mA} / \mathrm{cm}^{2}$, was used to transfer the pattern from the e-beam resist to the $\mathrm{Au}$ film. The a-C film was then etched through the Au mask using oxygen reactive ion etching (RIE). Steps with $\alpha \approx 60^{\circ}$ were defined by Ar ion milling perpendicular to the surface at $V=500 \mathrm{~V}$ and $J=0.5 \mathrm{~mA} / \mathrm{cm}^{2}$ with a rotation of the sample holder. Steps with $\alpha \approx 45^{\circ}$ were obtained by using an $\mathrm{Ar}$ ion beam oriented at $15^{\circ}$ with respect to the substrate normal, without rotating the sample holder. In both cases, after ion milling, the a-C residues were removed by oxygen RIE.

The YBCO film of 150-160 nm was deposited by laser ablation at an oxygen pressure of 0.6 mbar and a substrate temperature of $800^{\circ} \mathrm{C}$. It was in situ covered by a $200 \mathrm{~nm}$ thick $\mathrm{Au}$ film. The bilayer $\mathrm{YBCO} / \mathrm{Au}$ was etched through a photoresist mask using tilted $\mathrm{Ar}$ ion milling oriented at $60^{\circ}$ with respect to the substrate normal. Thus, microbridges with widths, $W$, between 2 and $8 \mu \mathrm{m}$ were defined, together with two YBCO stripes attached on both sides of the microbridge. Au bond pads were subsequently defined using a photoresist mask and Ar ion milling, oriented at an angle of $65^{\circ}$ with respect to the substrate normal in order to protect the YBCO stripes present along the step. For steps with $\alpha \approx 45^{\circ}$, microbridges were patterned by using perpendicular ion milling after the deposition of the YBCO film and of a thin layer of $\mathrm{Au}$ of $20 \mathrm{~nm}$. A thicker, $200 \mathrm{~nm}$, Au film was then deposited on the chip through a lift off mask 
which protected the microbridges and the electrodes. The Au was etched, using tilted Ar ion milling, and remained only on the step region being in contact with the microbridge. The Au bond pads, for this SEJs, were finally defined by using a lift off procedure through a photoresist mask. Each chip contains 20 devices. For both kinds of structures, it is possible to make four point measurements of the whole junction, of the YBCO stripes, of the $\mathrm{Au} / \mathrm{YBCO}$ interface, and of the top GB and bottom grain boundaries, by a proper choice of the contacts pads.

\section{MEASUREMENTS}

Current-voltage characteristics, $(I V c)$, were recorded as a function of the temperature, $T$, and of the external magnetic field, $H$, for the SEJs of different angles and, separately, of the top and bottom GBs, as well as of the YBCO stripes (or of the Au/YBCO interface). A detailed discussion of these measurements for SEJs on steeper steps $\left(\alpha \approx 60^{\circ}\right)$ is reported elsewhere [9]. Here we summarize the major results.

YBCO stripes which contacted both sides of the SEJs had a critical temperature, $T_{\mathrm{c}}^{\text {tripe }}$, between 60 and $85 \mathrm{~K}$. The critical current density, $J_{\mathrm{c}}^{\text {Stripe }}$, at $77 \mathrm{~K}$, was of the order of $10^{6} \mathrm{~A} / \mathrm{cm}^{2}$. The electrodes showed a $T_{\mathrm{c}}^{\text {film }}$ of $88 \mathrm{~K}$, the SEJs had, instead, a $T_{\mathrm{c}}^{\mathrm{SEJ}}$ varying between 70 and $80 \mathrm{~K}$. The $I_{\mathrm{c}} R_{\mathrm{N}}$ product of the junctions (where $I_{\mathrm{c}}$ is the critical supercurrent, and $R_{\mathrm{N}}$ is the normal resistance) were $0.2-3 \mathrm{mV}$ at $T=4.2 \mathrm{~K}$ and $0.02-0.1 \mathrm{mV}$ at $T=77 \mathrm{~K}$. A typical $I V c$ of the top GB of SEJ D1 is shown in Fig. 2 at $T=4.2 \mathrm{~K}$. It can be described by a RSJ model though an excess current is observed. The $I V c$ of the whole SEJ was identical to the $I V c$ of the top GB, within the measurement accuracy. The critical current of the bottom GB was larger than $5 \mathrm{~mA}$, in the range of temperature $T_{\mathrm{c}}^{\mathrm{Stripe}}<T<T_{\mathrm{c}}^{\mathrm{SEJ}}$, for a SEJ with a $T_{\mathrm{c}}^{\text {Stripe }}<T_{\mathrm{c}}^{\mathrm{SEJ}}$. The $I V c$ of the SEJ remained unchanged after the removal of the YBCO stripes. This was verified for more than 20 junctions on 4 different chips. As these SEJs consist of two well defined GBs [9] this proves that the weak link behaviour is determined by the top GB. The critical current of the top GB showed a strong response to the applied magnetic field. The $I_{\mathrm{c}}(H)$ dependence was generally symmetric with respect to the central peak though deviations from an ideal Fraunhofer pattern were generally observed. $I_{\mathrm{c}}$ oscillated with $H$, with a modulation depth of $30-50 \%$.

For devices fabricated on steps with $\alpha \approx 45^{\circ}$ the resistance $R_{\mathrm{s}}$ of the interface $\mathrm{Au} / \mathrm{YBCO}$ was about $1-2 \mathrm{~K} \Omega$, which corresponds to a specific interface resistance, $\rho_{\mathrm{s}}$, of the order of $10^{-6} \Omega$ $\mathrm{cm}^{2}$. The high value of $R_{\mathrm{s}}$ compared with the usual normal resistance $R_{\mathrm{N}}$ of a SEJ [10], generally no more than a few $\Omega$, indicates that, even when the Josephson junction is in its resistive state, almost all the current flows through it (only a very small percentage, of the order of $R_{\mathrm{N}} / R$ passes through the $\mathrm{Au} / \mathrm{YBCO}$ interface).

From transport measurements, performed on 10 junctions on the same chip, it was possible to group the behaviour of these SEJs in two categories, that we will refer to as type A and type B. For both, the $T_{\mathrm{c}}^{\mathrm{SEJ}}$ ranged between $60-70 \mathrm{~K}$. The lower value of $T_{\mathrm{c}}$ for these SEJs may be due to the different fabrication procedure.

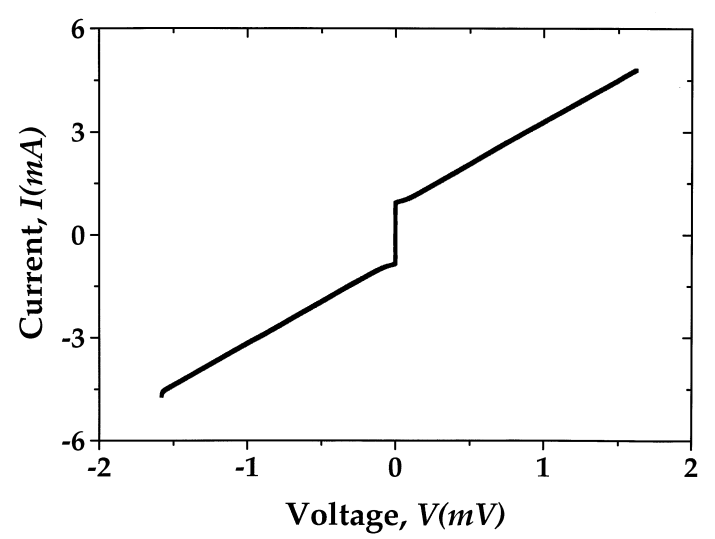

Fig. 2. Typical $I V$ curve of the top GB of a $8 \mu \mathrm{m}$ wide step edge junction $18 \mathrm{~A} 1$ at $T=4.2 \mathrm{~K}$. 
(a)

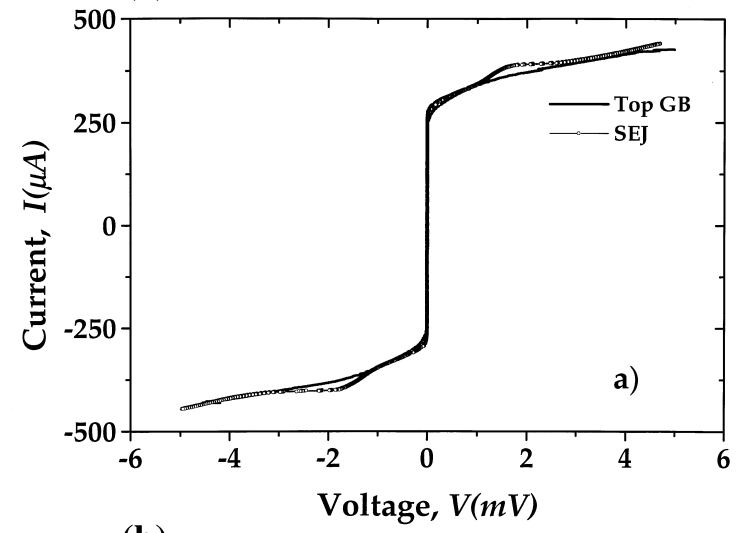

(b)

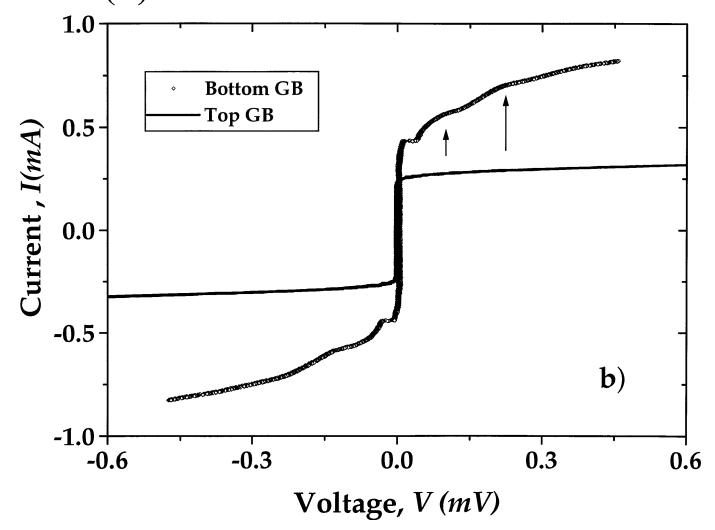

Fig. 3. $I V$ characteristics of a $2 \mu \mathrm{m}$ wide type A SEJ $25 \mathrm{C} 3$ at $T=4.2 \mathrm{~K}$ : (a) shows the $I V c$ of both the SEJ (continuous line with open circles) and the top GB (continuous line); (b) the $I V$ characteristics of both the top and bottom GB (open diamonds) are compared. The kinks present in the $I V c$ of the top are indicated by arrows.

Figure 3(a) shows the $I V c$ of the top GB and of the whole type A SEJ 25A3 at $T=4.2 \mathrm{~K}$. The two curves differ clearly. It is also evident that the $I_{\mathrm{c}}$ and $R_{\mathrm{N}}^{\mathrm{SEJ}}$ of the whole junction are mainly defined by the top GB. The $I V c$ of the top GB is compared with the $I V c$ of the bottom GB in Fig. 3(b). The $I V c$ of the bottom GB shows a typical RSJ shape at very low voltages $(40-50 \mu \mathrm{V})$ and the presence of two singularities, or "kinks", at finite voltage. By applying a weak external magnetic field $H$, the position in voltage of these kinks moved back and forth, by increasing $H$. Moreover, their shape could be strongly modulated by varying $H$. This suggests that the two kinks may correspond to the critical currents of weak links connected in series with the weakest one (which defines the critical current value in the $I V c$ of the bottom GB), each having a different cross section and critical current density. They are located below the top GB and are distributed along the step, possibly at the bottom edge of it. This statement is further confirmed by the $R_{\mathrm{N}}$ behaviour of the $I V c$ of the bottom GB, which increases for voltage above each kink (see Fig. 3). For all type A SEJs the $R_{\mathrm{N}}$ value at high voltage was less than a few $\Omega$, when the $I V c$ of the bottom GB was linear.

From Fig. 3(a) and (b), it is possible to observe that the $I V c$ of the top GB can be described by a flux flow model. Its shape and the high value of the normal resistance $\left(R_{\mathrm{N}}^{\mathrm{BGB}}=40 \Omega\right)$ appear, in fact, more typical of a flux flow regime. This is further confirmed by the fact that a very small modulation is observed in the presence of an external magnetic field of the order of a few mT. All type A $I V c$ s of the top GB showed the same shape with a rather high value of $R_{\mathrm{N}}$, between 10 and $40 \Omega$.

Figure 4 shows the $I V c$ of the bottom GB of the type B SEJ 25C1. It shows almost the same characteristics as the bottom GB of type A junctions, with a typical RSJ shape at very low voltages and a presence of kinks at higher voltage. In this case, however, the $I V c$ of the whole SEJ 


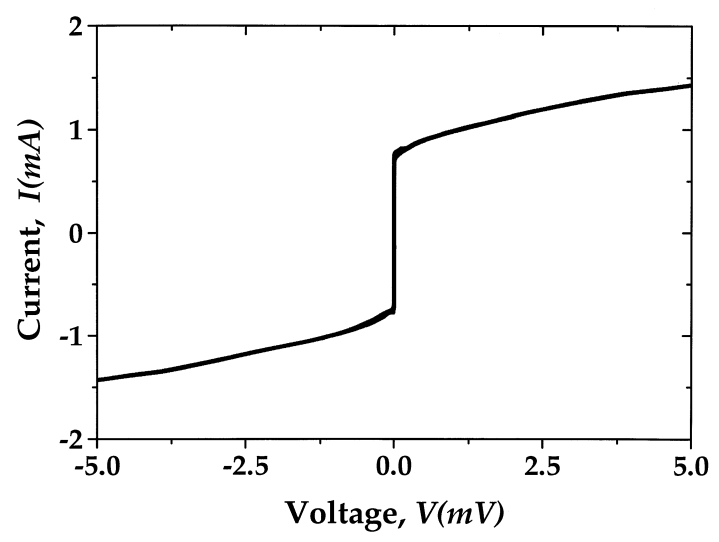

Fig. 4. $I V$ curve of the bottom GB of a $4 \mu \mathrm{m}$ wide type B SEJ $25 \mathrm{~B} 1$ at $T=4.2 \mathrm{~K}$.

was identical to the $I V c$ of the bottom GB. In the whole range of temperatures between 4.2 and $70 \mathrm{~K}$ (the last corresponds to $T_{\mathrm{c}}^{\mathrm{SEJ}}$ ) the $I_{\mathrm{c}}$ of the top GB exceeded $5 \mathrm{~mA}$. The top GB appears to behave as a superconductive short, for a type B SEJ, while a series of week links is present along the step.

\section{DISCUSSION}

The data presented in the previous section show that the transport properties of SEJs are strictly related to the step angle. For a step angle $\alpha \approx 60^{\circ}$, we find that the weak link behaviour of the SEJ is determined by one GB, namely the top GB, located at the top edge of the step. A closer inspection of the microscopic structural properties of these junctions revealed, in fact, that the two GBs are very dissimilar. While the top GB is a symmetric grain boundary type, with a small part of basal plane faced tilted grain boundary, the bottom GB is a pure BPGB (see Fig. 5). This allows one to attribute the Josephson behaviour of the SEJ to the presence of the SGB component in the top GB. As reported in Ref. [9] the maximum modulation depth of the $I_{\mathrm{c}}(H)$ of the top GB can be related to the SGB part and the background current to the BPGB part. This makes it possible to estimate the critical current density $J_{\mathrm{c}}$ of the BPGB to be of the order of $10^{6} \mathrm{~A} / \mathrm{cm}^{2}$ at $4.2 \mathrm{~K}$.

From the transport measurements on SEJs with a step $\alpha \approx 45^{\circ}$ we can correlate the multiple weak links with multiple GBs distributed along the step and at the bottom edge of it, for both type A and B. This was also reported by Herrmann et al. [11] for SEJs with the same value of

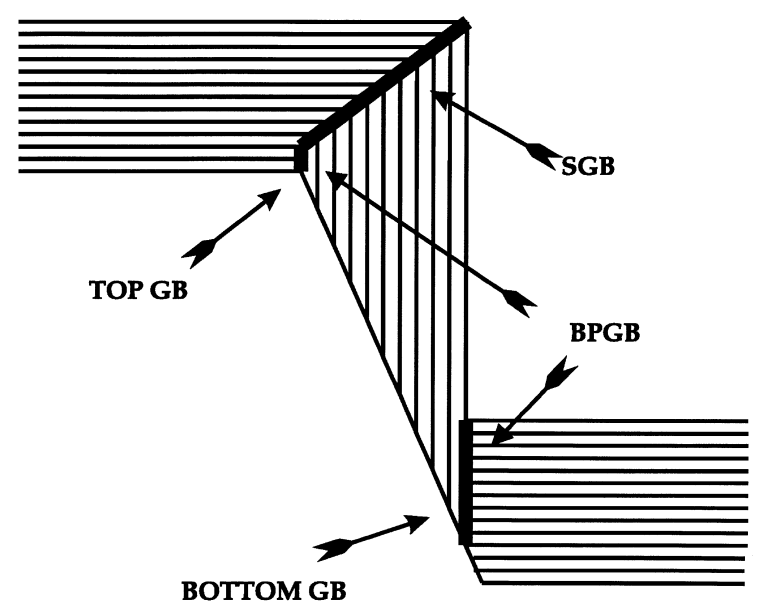

Fig. 5. Schematic representation of the GBs formed at the top and at the bottom edges of a $60^{\circ}$ step [9]. The ratio between the symmetric GB and the basal plane faced tilted GB in the top GB is almost in scale. It is worth noticing that the bottom GB does not nucleate at the bottom edge of the step. The critical current density of the SGB is much smaller than the one of the BPGB. 
the step angle. Results relative to SEJs on more steep steps allows one to speculate that these GBs should mostly consist of a SGB type. The transport properties of the top GB may, instead, reveal that either it is not situated exactly at the top edge of the step or, when this is not the case, it is mostly formed by BPGB with an effective area much reduced with respect to the thickness of the YBCO film. The first consideration would, in fact, justify the superconductive short observed in the top GB of type B junctions, while the second is able to explain the low value of the critical current of the top GB, of type A junctions. As the estimated $J_{\mathrm{c}}^{\mathrm{BPGB}}$ value is about $10^{6} \mathrm{~A} / \mathrm{cm}^{2}$ at $T=4.2 \mathrm{~K}$ the effective area of the BPGB in the top GB should be 10 $20 \mathrm{~nm}$, in order to get a critical current of the order of $0.5 \mathrm{~mA}$ [see Fig. 3(a), (b)]. We tend, however, to exclude the possibility that the top GB consists of a BPGB with the same thickness of the film, which could equally well explain the behaviour of type B junctions. To our knowledge, there is no structural investigation which is able to justify this statement. A clearer understanding of the transport properties of these SEJs requires a detailed microscopic investigation of the structural properties of the GBs. Such an analysis is presently in progress.

\section{CONCLUSION}

Transport properties were studied for two step angles, $\alpha \approx 60^{\circ}$ and $\alpha \approx 45^{\circ}$. Junctions on the steeper steps, $\alpha \approx 60^{\circ}$, were the most reliable, and similar performances were registered for several junctions on the same chip and on different chips.

Electron microscopy has previously shown that there are two dominating grain boundaries in step edge junctions with a steep angle [5,6]. High resolution transmission electron microscopy of our samples showed that the top GB consisted mainly of a symmetric part with a small component of BPGB. The bottom GB, on the other hand, was purely of a BPGB nature.

From comparative measurements using the contact pads on the stripes attached to the middle part of the step edge junction, we conclude that the top GB is the one that gives the weak link, Josephson character to the combined junction. The SGB part acts as a weak coupling link while the coupling is stronger in the BPGB part with $J_{\mathrm{c}}^{\mathrm{BPGB}} \approx 10^{6} \mathrm{~A} / \mathrm{cm}^{2}$ at $T=4.2 \mathrm{~K}$.

The spread in parameter of step-edge junctions can be related to the presence, in the top GB, of the BPGB component, with a variable thickness. As the $J_{\mathrm{c}}^{\mathrm{BPGB}}$ is high, the different amount will strongly affect the critical current value of the top GB and its modulation depth. On the other hand, the absence of a Josephson behaviour in the BPGB part, makes the step edge junctions interesting for applications, such as superconducting quantum interference devices (SQUID)s, in which only one junction is desired.

The transport properties of junctions on $45^{\circ}$ steps appear to be more strongly affected by the local shape of the edge of the step and eventually by the presence of defects. We could distinguish two different behaviours. Type A junctions showed a highly resistive top GB and a series of GBs, acting as weak links, distributed along the step profile and at the bottom edge of the step. Type B junctions, on the other end, showed a superconductive short at the top edge and, again, a series of GBs along the step. The lack of reproducibility of low angle step edge junctions can be related the nucleation of several GBs mostly of the symmetric type, along the step profile.

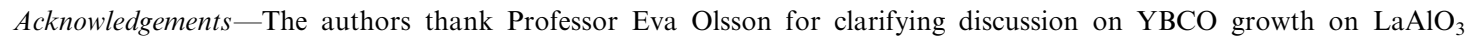
steps. One of us, F. Lombardi, would like to thank Dr F. Tafuri and Dr A. Tzalenchuk for valuable comments. This work has been partially supported by the EU HCM program contract no. CHRX-CT94-0523 and the Swedish Consortium on Superconductivity.

\section{REFERENCES}

1. A. Barone and G. Paterno. 1982. in Physics and Applications of the Josephson effect. ed. Wiley.

2. R. Gross and P. Chaudary. 1992. in Principle and Applications of Superconductive Quantum Interference Devices. ed. A. Barone. World Scientific, New York. pp. 419.

3. M. Gustafsson, E. Olsson, H.R. Yi, D. Winkler and T. Claeson, Nucleation and growth of YBCO on wavy step edges in (001) $\mathrm{LaAlO}_{3}$, J. All. Comp. 251, 19 (1997).

4. C.L. Jia, B. Kabius, K. Urban, K. Herrman, G.J. Cui, J. Schubert, W. Zander, A.I. Braginski and C. Heiden, Microstructures of epitaxial YBCO films on step-edge $\mathrm{SrTiO}_{3}$ substrates, Physica C 175, 545 (1991).

5. C.L. Jia, B. Kabius, K. Urban, K. Herrman, J. Schubert, W. Zander and A.I. Braginski, The microstructure of epitaxial YBCO films on steep steps in $\mathrm{LaAlO}_{3}$, Physica C 196, 221 (1992). 
6. M. Gustafsson, E. Olsson, H.R. Yi, D. Winkler and T. Claeson, Grain boundary evolution of YBCO in the vicinity of steps on patterned (001) $\mathrm{LaAlO}_{3}$, Appl. Phys Lett. 70(21), 2903 (1997).

7. M. Grove, R. Dittmann, M. Bode, M. Siegel and A.I. Braginski, Current transport across YBCO-Au interfaces, Appl. Phys. Lett. 69(5), 696 (1996).

8. I. Iguchi, K. Nukui and K. Lee, Dynamic Cooper pairing by tunnel injection of quasiparticles into high- $T_{c} Y B C O$ superconductor, Phys. Rev. B 50(1), 457 (1994).

9. F. Lombardi, Z.G. Ivanov, G.M. Fischer, E. Olsson and T. Claeson, Transport and structural properties of the top and the bottom grain boundaries in YBCO step-edge junction, Appl. Phys. Lett. 72(2), 249 (1998).

10. H.R. Yi, Z.G. Ivanov, D. Winkler, Y.M. Zhang, H. Olin, P. Larsson and T. Claeson, Improved step edge on $\mathrm{LaAlO}_{3}$ substrates by using amorphous carbon etch masks, Appl. Phys. Lett. 65(9), 1117 (1996).

11. K. Herrmann, G. Kundel, M. Siegel, J. Schubert, W. Zander, A.I. Braginski, C.L. Jia, B. Kabius and K. Urban, Correlation of YBCO step edge junction characteristics with microstructure, J. Appl. Phys. 78(2), 1131 (1995). 